\title{
DOMAIN WORD TRANSLATION BY SPACE-FREQUENCY ANALYSIS OF CONTEXT LENGTH HISTOGRAMS
}

\author{
Pascale Fung \\ Computer Science Department \\ Columbia University \\ New York, NY 10027 \\ pascale@cs.columbia.edu
}

\begin{abstract}
We report a new statistical feature relating a bilingual word pair in a non-parallel English-Chinese corpus. It is found that the lengths of context segments of a word is closely correlated to that of its translation, even when the corpus is non-parallel, i.e., monolingual texts which are not translations of each other. The context segment length histogram of a word has a characteristic pattern and corresponds to that of its translation. If a word appears most frequently in long segments, its translation is found to be most likely occurring in long segments. One way to match these histograms is to first extract their salient shape characteristics by space-frequency analysis and then match them against each other using dynamic time warping. The results of matching can be used in combination with other statistical features to bootstrap a word or term translation algorithm from non-parallel corpora.
\end{abstract}

\section{INTRODUCTION}

Translating domain-specific words is a significant component in machine translation and machine-aided translation systems. These words are often not found in standard dictionaries. Human translators, not being experts in every technical or regional domain, cannot produce their translations effectively. Automatic translation of words in specific domains is therefore highly desirable.

One approach to obtaining domain-specific word translations employs statistical learning algorithms to automatically extract a lexicon from large parallel bilingual texts which contain the same material in two translations $[1,3,4,5,7]$. The weakness of this approach is that parallel texts are relatively scarce. However, non-parallel texts can be found easily, since they are simply monolingual texts concerning the same domain but not necessarily translations. Another weakness of some previous approaches is their orientation toward European language pairs. They cannot be applied to language pairs such as Chinese and English. A new approach is which would be extendable to other language pairs is needed.

This paper demonstrates a pattern matching method by using a statistical feature, the context length histogram, to correlate pairs of translated words. It will also be shown how space-frequency analysis is used for matching such word pair signals for translation.

As input corpus, the bilingual transcription of the Hong Kong Legislative Council debates is used for experiments [6]. The data is from 1988-1992, with the first 73618 sentences from the English text, and the next 73618 sentences from the Chinese text. There are no overlapping sentences between the texts. The topics of these debates focus on the political and social issues of Hong Kong.

\section{ALGORITHM OVERVIEW}

The procedure for our algorithm is as follows:

1 Segment both the English and the Chinese texts by delimiters

2 Compute segment lengths of both texts

3 Compute the context length histograms for all words

Transform the histograms using space-frequency analysis

Dynamic time warping to match the transformed graphs

6 Obtain bilingual word pairs from matching results

\subsection{Segments of texts in English and Chinese}

Segmental information was found to be useful in providing statistics for word pair matching. In parallel corpora, a long sentence in one language would correspond to a long sentence in its translation to another language. Such information could be used to align sentences and word pair matching could be carried out 
from aligned sentence pairs $[1,4,5,7]$. Texts in noisy parallel corpora could be segmented by word pair anchor points [3] and aligned. In a non-parallel bilingual text, there is no such linear sentence or segmental mapping - given any sentence in one language, its translation does not even appear in the other text. We need to find segmental correspondence which are textindependent.

It is generally found that English sentences, delimited by a full-stop(period), are shorter than Chinese sentences, delimited by a round circle. Very often, Chinese would use commas or semi-colons instead where in English a full-stop would have been used. Therefore, full-stops in English and Chinese are not good corresponding delimiters for segments. On the other hand, punctuations in general are still good delimiters. So we divided both the English and the Chinese texts into segments delimited by one of the following punctuations: an English full-stop, a Chinese full-stop, a comma, a question mark, a semi-colon or an exclamation mark.

We postulate that if a word appears frequently in short segments, then its translation would also appear more frequently in short segments. For example, the word figure is often seen in segments like "We will show this in figure 1", "The ... is shown as follows in figure 1 " etc. It rarely appears in long segments. Its translation is used in the same ways in Chinese. We define the length of an English segment to be the number of words in that segment. However, the length of a Chinese segment is defined as the number of characters in that segment to compensate for its linguistic difference with English.

\section{HISTOGRAMS OF CONTEXT SEGMENT LENGTHS}

Next, we compute the histogram of context segment lengths for each word in English and Chinese, assuming the maximum segment length is 100 and the minimum is one ${ }^{1}$. This is also the range for the $x$-axis for the histogram plot. For Government, part of its concordance in the English text is shown in Figure 1, one segment per line. The concordance for 政府, the Chinese word is shown in Figure 2. The first field indicates the length of each segment. The $y$ value of the histogram indicates how many times Government occurs in a segment of length $x$. Since this information would be used to match words in non-parallel texts with very different occurrence frequencies, we normalize this graph so that the total area under the graph would be one. We

\footnotetext{
${ }^{1}$ In actual case, the maximum is usually around 70
}

Figure 1: Part of the concordance for Government in English

\begin{tabular}{|c|c|}
\hline length & concordance \\
\hline 20 & $\begin{array}{l}\text { council has brought with it a greater diversity } \\
\text { of views and a closer scrutiny of the work of } \\
\text { the Government }\end{array}$ \\
\hline 23 & $\begin{array}{l}\text { The policies of the Government which I shall } \\
\text { put before you this afternoon will require } \\
\text { a great deal of work from the Administration }\end{array}$ \\
\hline 18 & $\begin{array}{l}\text { The Government have already taken a number } \\
\text { of measures to try to reduce the size of } \\
\text { the problem }\end{array}$ \\
\hline 20 & $\begin{array}{l}\text { There have been calls for the Government to } \\
\text { change its policy to allow contractors to } \\
\text { import workers for specific projects }\end{array}$ \\
\hline 30 & $\begin{array}{l}\text { And it continues actively to look for } \\
\text { opportunities to provide services through } \\
\text { bodies outside the } \\
\text { Government where there are clear advantages } \\
\text { in terms of cost - effectiveness and } \\
\text { management flexibility }\end{array}$ \\
\hline 12 & $\begin{array}{l}\text { A number of major facilities are currently } \\
\text { being built by the Government }\end{array}$ \\
\hline
\end{tabular}

Figure 2: Part of the concordance for Government in Chinese

\begin{tabular}{|c|c|}
\hline length & concordance \\
\hline 7 & 政府 以往 卻認為 \\
\hline 15 & 我 希望 政府 盡快 提出一套 改善 措施 \\
\hline 25 & $\begin{array}{l}\text { 而且 政府 可能 需要 更 嚴原 地 執行 } \\
\text { 多年 前 與 兩 巴訂立的 協議 }\end{array}$ \\
\hline 14 & 我並不反對政府批准 交通 專利 權 \\
\hline 27 & $\begin{array}{l}\text { 惟 政府 必須能豿 對每 間 專利 } \\
\text { 公司 進行 全面而有效 的監察 與 管制 }\end{array}$ \\
\hline 18 & $\begin{array}{l}\text { 請 政府將兩巴的運作 和加 價 問題 全面 } \\
\text { 檢討 }\end{array}$ \\
\hline 29 & $\begin{array}{l}\text { 這個制度 令 政府 能夠 有效地 管理 } \\
\text { 各種 互相 競爭 交通工具 的 日常 運作 }\end{array}$ \\
\hline
\end{tabular}

obtain a graph of the same shape but with different $y$ values.

Example plots of the words Government and debate in both languages are shown in Figures 4 and 6. Note the visual similarity between the two graphs of a word pair. By inspecting the original corpus, we found that the salient narrow peaks such as the one at $x=4$ in the histogram for Government and those at $x=10$ and then 16 for debate are caused by the predominance of domain-specific rigid phrases of those segment lengths. 


\section{SPACE-FREQUENCY TRANSFORMATION FOR MATCHING HISTOGRAMS}

From the histogram figures, we can see that in general, the similarity between the histogram of a word in English and that of its translation in Chinese have similarities perceivable to the human eyes, i.e., we can see they have similar shapes. To match these shapes algorithmically, however, is much more difficult. Thus, we need a way to analyse the general hump, as well as the peaks and valleys of the plots, preserving the order in which they appear.

A space-frequency transformation can be used to analyze the signals in order to emphasize their characteristics and to reduce superfluous information. The difference of two Gaussians was used as a basis function (Figure 3):

$$
\begin{aligned}
h & =\left(\frac{1}{\sqrt{2 \pi} a 2} e^{-0.5 u^{2} / a 2^{2}}\right)-\left(\frac{1}{\sqrt{2 \pi} a} e^{-0.5 u^{2} / a^{2}}\right) \\
a & =1,5,10, \ldots, N \\
a 2 & =0.5 a \\
u & =-5 a: 5 a
\end{aligned}
$$

The total area of the basis function is zero. Different $a$ would contract or dilate the basis function, thereby changing the window size of the transformation. The

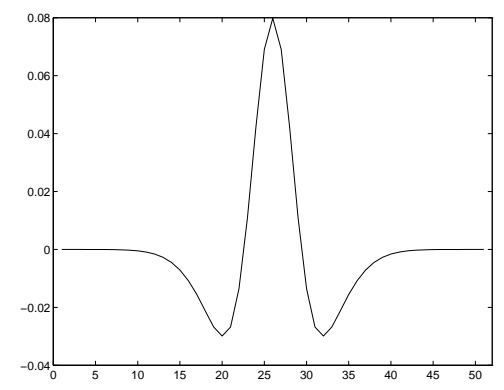

Figure 3: Difference of two Gaussians as the basis function

basis functions was convolved with the interpolated graph of the original signal $V$ at all positions on the $x$-axis

$$
\operatorname{val}\left(V 1_{i}[j]\right)=\int_{0}^{N} h \cdot V^{\prime}
$$

After transformation, the value $i$ at $(x, y)$ would denote the intensity at frequency $y$ at point $x$. At any given $x$, the plot is a weighted combination of different space-frequency basis functions at frequencies marked by the $y$-axis. The weight is shown by $i$. The spacefrequency transformation provides us an analytical way of looking at the histogram signals. By quantizing the signals, the relative peaks and valleys on the signals become more salient.

Looking at the left plot in Figure 5, the transformation plot of the word Government in English, we see that there is a small white patch at around $y=5$, high frequency. This corresponds to the sharp peak, a local maxima in the original histogram at around $x=5$. The bigger white patch at lower frequencies and at around $x=28$ corresponds to the general shape of the original signal having a gentle hump there. There are corresponding sharp peaks and a gentle hump in the signals for the Chinese word, and in its transformation figure.

\section{DTW MATCHING}

After transformation, the signals of word pairs are more or less warped versions of each other in the $\mathrm{x}$-axis. To match the transformed graphs, we use dynamic time warping (DTW) on the difference of the intensity at each frequency. At each $y$, the $(x-1)$-dimensional row vector is the delta encoder of the original $x$-dimensional vector. We compare the row vector of a word $V 1$ to that of another word $V 2$ at the same $y_{i}$ value, giving a score $D T W\left(V 1, V 2, y_{i}\right)$. The total correlation score between two graphs is $\sum_{i=1}^{N} \operatorname{DTW}\left(V 1, V 2, y_{i}\right)$ where $\operatorname{DTW}\left(V 1, V 2, y_{i}\right)$ is the DTW score of the two delta vectors in frequency band $i$.

\section{RESULT AND DISCUSSION}

We have shown a novel algorithm for extracting bilingual word pairs from same domain, non-parallel texts of Chinese and English. The signal representation of word features ensures that this algorithm is robust to language groups. DTW is an effective matching function on the space-frequency transformations of the word signals. We have tested this algorithm on more than 50 word pairs, the result shows that about 40 of the words match most closely to their translations in the other language. We will combine this feature with other statistical information found in our previous work [2] in order to improve the performance.

\section{ACKNOWLEDGMENT}

I wish to thank Truong-Thao Nguyen and other faculties at the Dept. of Electrical \& Electronic Engineering in HKUST for useful comments, Kathleen McKeown, Shree Nayar, and Hiroshi Murase for support and encouragement. 


\section{REFERENCES}

[1] Ido Dagan, Kenneth W. Church, and William A. Gale. Robust bilingual word alignment for machine aided translation. In Proceedings of the Workshop on Very Large Corpora: Academic and Industrial Perspectives, pages 1-8, Columbus, Ohio, June 1993.

[2] Pascale Fung. Context similarity measure for building a bilingual dictionary from a non-parallel English-Chinese corpus. In Proceedings of the Third Annual Workshop on Very Large Corpora, pages 173-183, Boston, Massachusettes, 1995.

[3] Pascale Fung. A pattern matching method for finding noun and proper noun translations from noisy parallel corpora. In Proceedings of the 33rd Annual Conference of the Association for Computational Linguistics, pages 236-233, Boston, Massachusettes, 1995.

[4] Julian Kupiec. An algorithm for finding noun phrase correspondences in bilingual corpora. In Proceedings of the 31st Annual Conference of the Association for Computational Linguistics, pages 17-22, Columbus, Ohio, June 1993.

[5] Frank Smadja and Kathleen McKeown. Translating collocations for use in bilingual lexicons. In Proceedings of the ARPA Human Language Technology Workshop 94, Plainsboro, New Jersey, June 1994.

[6] Dekai Wu. Aligning a parallel English-Chinese corpus statistically with lexical criteria. In Proceedings of the 32nd Annual Conference of the Association for Computational Linguistics, pages 80-87, Las Cruces, New Mexico, June 1994.

[7] Dekai Wu and Xuanyin Xia. Learning an EnglishChinese lexicon from a parallel corpus. In Proceedings of the First Conference of the Association for Machine Translation in the Americas, pages 206213, Columbia, Maryland, 1994.
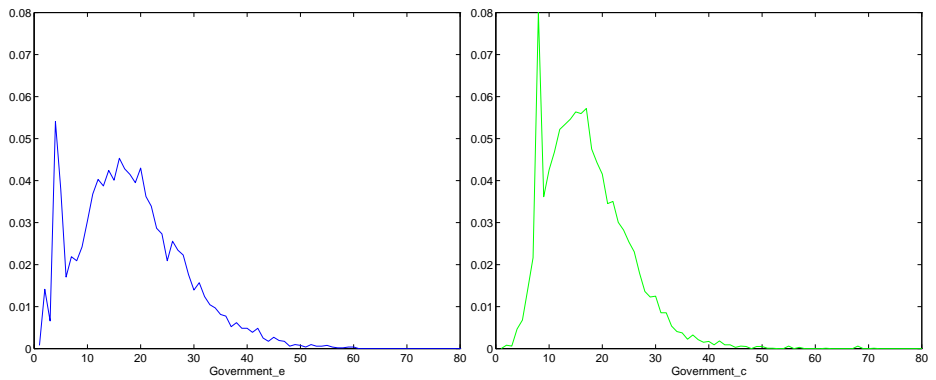

Figure 4: Normalized histogram of Government in English and Chinese
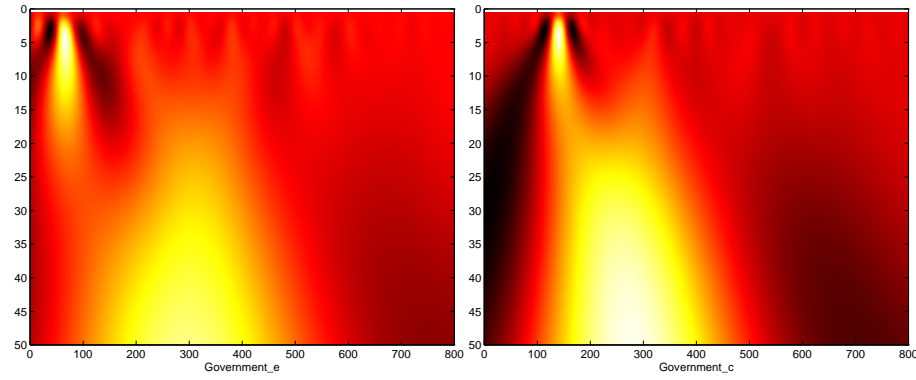

Figure 5: Space-frequency plots of Government in English and Chinese



Figure 6: Normalized histogram of debate in English and Chinese
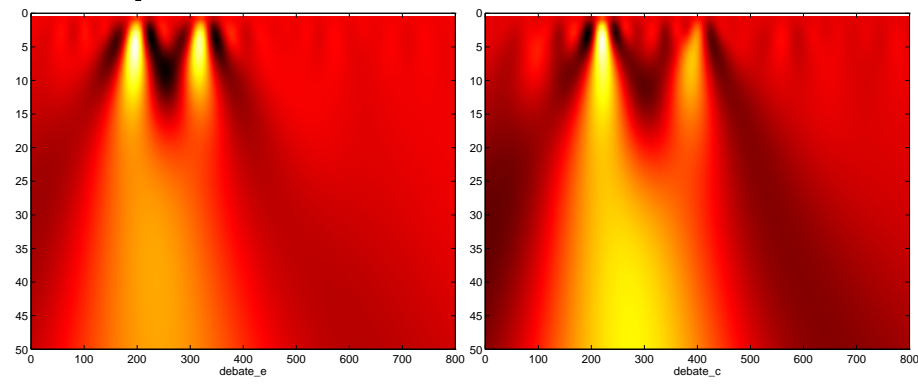

Figure 7: Space-frequency plots of debate in English and Chinese 